\title{
ON RESOLVING EDGE COLORINGS IN GRAPHS
}

\author{
VARAPORN SAENPHOLPHAT and PING ZHANG
}

Received 26 November 2002

\begin{abstract}
We study the relationships between the resolving edge chromatic number and other graphical parameters and provide bounds for the resolving edge chromatic number of a connected graph.
\end{abstract}

2000 Mathematics Subject Classification: 05C12, 05C15.

1. Introduction. For edges $e$ and $f$ in a connected graph $G$, the distance $d(e, f)$ between $e$ and $f$ is the minimum nonnegative integer $a$ for which there exists a sequence $e=e_{0}, e_{1}, \ldots, e_{a}=f$ of edges of $G$ such that $e_{i}$ and $e_{i+1}$ are adjacent for $i=0,1, \ldots, a-1$. For an edge $e$ of $G$ and a subgraph $F$ of positive size in $G$, the distance between $e$ and $F$ is defined as

$$
d(e, F)=\min \{d(e, f): f \in E(F)\} .
$$

A decomposition of a graph $G$ is a collection of subgraphs of $G$, none of which have isolated vertices, whose edge sets provide a partition of $E(G)$. A decomposition of $G$ into $k$ subgraphs is a $k$-decomposition. A decomposition $\mathscr{D}=\left\{G_{1}, G_{2}, \ldots, G_{k}\right\}$ is ordered if the ordering $\left(G_{1}, G_{2}, \ldots, G_{k}\right)$ has been imposed on $\mathscr{D}$. For an ordered $k$-decomposition $\mathscr{D}=\left\{G_{1}, G_{2}, \ldots, G_{k}\right\}$ of a connected graph $G$ and $e \in E(G)$, the $\mathscr{D}$-code (or simply the code) of $e$ is the $k$-vector

$$
c_{\mathscr{D}}(e)=\left(d\left(e, G_{1}\right), d\left(e, G_{2}\right), \ldots, d\left(e, G_{k}\right)\right) .
$$

Hence exactly one coordinate of $c_{\mathscr{D}}(e)$ is 0 , namely the $i$ th coordinate if $e \in$ $E\left(G_{i}\right)$. In [3], a decomposition $\mathscr{D}$ is defined to be a resolving decomposition for $G$ if every two distinct edges of $G$ have distinct $D$-codes. The minimum $k$ for which $G$ has a resolving $k$-decomposition is its decomposition dimension $\operatorname{dim}_{d}(G)$. A resolving decomposition of $G$ with $\operatorname{dim}_{d}(G)$ elements is a minimum resolving decomposition for $G$.

A resolving decomposition $\mathscr{D}=\left\{G_{1}, G_{2}, \ldots, G_{k}\right\}$ of a connected graph $G$ is defined in [5] to be independent if $E\left(G_{i}\right)$ is independent for each $i$ ( $1 \leq i \leq k$ ) in $G$. This concept can be considered from an edge-coloring point of view. Recall that a proper edge coloring (or simply, an edge coloring) of a nonempty graph $G$ is an assignment $c$ of colors (positive integers) to the edges of $G$ so that adjacent edges are colored differently, that is, $c: E(G) \rightarrow \mathbb{N}$ is a mapping 
such that $c(e) \neq c(f)$ if $e$ and $f$ are adjacent edges of $G$. The minimum $k$ for which there is an edge coloring of $G$ using $k$ distinct colors is called the edge chromatic number $\chi_{e}(G)$ of $G$. If $\mathscr{D}=\left\{G_{1}, G_{2}, \ldots, G_{k}\right\}$ is an independent decomposition of a graph $G$, then by assigning color $i$ to all edges in $G_{i}$ for each $i$ with $1 \leq i \leq k$, we obtain an edge coloring of $G$ using $k$ distinct colors. On the other hand, if $c$ is an edge coloring of a connected graph $G$, using the colors $1,2, \ldots, k$ for some positive integer $k$, then $c(e) \neq c(f)$ for adjacent edges $e$ and $f$ in $G$. Equivalently, $c$ produces a decomposition $\mathscr{D}$ of $E(G)$ into color classes (independent sets) $C_{1}, C_{2}, \ldots, C_{k}$, where the edges of $C_{i}$ are colored $i$ for $1 \leq i \leq k$. Thus, for an edge $e$ in a graph $G$, the $k$-vector

$$
c_{\mathscr{D}}(e)=\left(d\left(e, C_{1}\right), d\left(e, C_{2}\right), \ldots, d\left(e, C_{k}\right)\right)
$$

is called the color code (or simply the code) $\mathcal{C}_{\mathscr{D}}(e)$ of $e$. If distinct edges of $G$ have distinct color codes, then $c$ is called a resolving edge coloring (or independent resolving decomposition) of $G$ in [5]. Thus a resolving edge coloring of $G$ is an edge coloring that distinguishes all edges of $G$ in terms of their distances from the resulting color classes. A minimum resolving edge coloring uses a minimum number of colors, and this number is the resolving edge chromatic number $\chi_{r e}(G)$ of $G$. Since every resolving edge coloring is an edge coloring and every resolving edge coloring is a resolving decomposition, it follows that

$$
2 \leq \max \left\{\operatorname{dim}_{d}(G), \chi_{e}(G)\right\} \leq \chi_{r e}(G) \leq m
$$

for each connected graph $G$ of size $m \geq 2$.

To illustrate these concepts, consider the graph $G$ of Figure 1.1. Let $\mathscr{D}_{1}=$ $\left\{G_{1}, G_{2}, G_{3}\right\}$ be the decomposition of $G$, where $E\left(G_{1}\right)=\left\{v_{1} v_{2}, v_{2} v_{5}\right\}, E\left(G_{2}\right)=$ $\left\{v_{2} v_{3}, v_{2} v_{6}, v_{3} v_{6}\right\}$, and $E\left(G_{3}\right)=\left\{v_{3} v_{4}, v_{3} v_{5}\right\}$. Since $\mathscr{D}_{1}$ is a minimum resolving decomposition of $G$, it follows that $\operatorname{dim}_{d}(G)=3$. Define an edge coloring $c$ of $G$ by assigning the color 1 to $v_{1} v_{2}$ and $v_{3} v_{5}$, the color 2 to $v_{2} v_{5}$ and $v_{3} v_{6}$, the color 3 to $v_{2} v_{3}$, and the color 4 to $v_{2} v_{6}$ and $v_{3} v_{4}$ (see Figure 1.1(b)). Since $c$ is a minimum edge coloring of $G$, it follows that $\chi_{e}(G)=4$. However, $c$ is not a resolving edge coloring. To see that, let $\mathscr{D}_{2}=\left\{C_{1}, C_{2}, C_{3}, C_{4}\right\}$ be the decomposition of $G$ into color classes resulting from $c$, where the edges in $C_{i}$ are colored $i$ by $c$. Then $\mathcal{C}_{\Phi_{2}}\left(v_{2} v_{5}\right)=(1,0,1,1)=\mathcal{C}_{\mathscr{D}_{2}}\left(v_{3} v_{6}\right)$. On the other hand, define an edge coloring $c^{*}$ of $G$ by assigning the color 1 to $v_{1} v_{2}$ and $v_{3} v_{5}$, the color 2 to $v_{2} v_{3}$, the color 3 to $v_{2} v_{5}$ and $v_{3} v_{4}$, the color 4 to $v_{2} v_{6}$, and the color 5 to $v_{3} v_{6}$ (see Figure 1.1(c)). Let $D^{*}=\left\{C_{1}, C_{2}, \ldots, C_{5}\right\}$ be the decomposition of $G$ into color classes of $c^{*}$. Then

$$
\begin{array}{rr}
\mathcal{C}_{\mathscr{D} *}\left(v_{1} v_{2}\right)=(0,1,1,1,2), & \mathcal{C}_{\mathscr{D} *}\left(v_{2} v_{3}\right)=(1,0,1,1,1), \\
\mathcal{C}_{\mathscr{D} *}\left(v_{2} v_{5}\right)=(1,1,0,1,2), & \mathcal{C}_{\mathscr{D} *}\left(v_{2} v_{6}\right)=(1,1,1,0,1), \\
\mathcal{C}_{\mathscr{D} *}\left(v_{3} v_{4}\right)=(1,1,0,2,1), & \mathcal{C}_{\mathscr{D} *}\left(v_{3} v_{5}\right)=(0,1,1,2,1), \\
\mathcal{C}_{\mathscr{D} *}\left(v_{3} v_{6}\right)=(1,1,1,1,0) .
\end{array}
$$




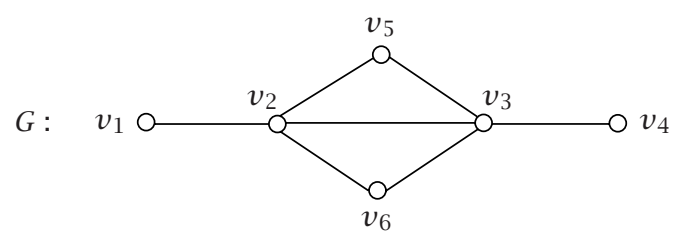

(a)

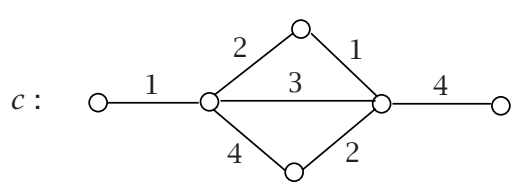

(b)

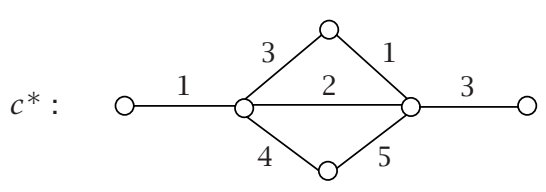

(c)

Figure 1.1. A graph $G$ with $\operatorname{dim}_{d}(G)=3, \chi_{e}(G)=4$, and $\chi_{r e}(G)=5$.

Since the $D^{*}$-codes of the edges of $G$ are all distinct, it follows that $c^{*}$ is a resolving edge coloring. Moreover, $G$ has no resolving edge coloring with 4 colors and so $\chi_{r e}(G)=5$.

The concept of resolvability in graphs has previously appeared in [7, 11, 12]. Slater $[11,12]$ introduced this concept and motivated by its application to the placement of a minimum number of sonar detecting devices in a network so that the position of every vertex in the network can be uniquely determined in terms of its distance from the set of devices. Harary and Melter [7] discovered these concepts independently as well. Resolving decompositions in graphs were introduced and studied in [3] and further studied in [6]. Resolving decompositions with prescribed properties have been studied in [5, 9, 10]. Resolving concepts were studied from the point of view of graph colorings in $[1,2]$. We refer to [4] for graph theory notation and terminology not described here.

In [5], all nontrivial connected graphs of size $m$ with resolving edge chromatic number 3 or $m$ are characterized. Also, bounds have been established for $\chi_{r e}(G)$ of a connected graph $G$ in terms of its size, diameter, or girth, as stated below.

THEOREM 1.1. If $G$ is a connected graph of size $m \geq 3$ and diameter $d$, then

$$
2 \leq \chi_{r e}(G) \leq m-d+3 .
$$

Moreover, $\chi_{r e}(G)=2$ if and only if $G=P_{3}$, and $\chi_{r e}(G)=m-d+3$ if and only if $G=P_{n}$ for $n \geq 4$. 
THEOREM 1.2. If $G$ is a connected graph of size $m$ and girth $\ell$, where $m \geq$ $\ell \geq 3$, then

$$
\chi_{r e}(G) \leq m-\ell+4
$$

Moreover, $\chi_{r e}(G)=m-\ell+4$ if and only if $G=C_{n}$ for some even $n \geq 4$.

In this paper, we study the relationships among the resolving edge chromatic number, edge chromatic number, and decomposition dimension of a connected graph, and provide bounds for the resolving edge chromatic number of a connected graph in terms of other graphical parameters in Section 2. We investigate the resolving edge colorings of trees in Section 3.

2. Bounds for resolving edge chromatic numbers. In this section, we establish bounds for the resolving edge chromatic number of a connected graph in terms of (1) its order and edge chromatic number; (2) its decomposition dimension and edge chromatic number. In order to this, we need some additional definitions and preliminary results. Let $\mathscr{D}$ be a decomposition of a connected graph $G$. Then a decomposition $\mathscr{D}^{*}$ of $G$ is called a refinement of $\mathscr{D}$ if every element in $\mathscr{D}^{*}$ is a subgraph of some element of $\mathscr{D}$. First, we present two lemmas, the first of which appears in [9].

LEMMA 2.1. Let $\mathscr{D}$ be a resolving decomposition of a connected graph $G$. If $\mathscr{D}^{*}$ is a refinement of $\mathscr{D}$, then $\mathscr{D}^{*}$ is also a resolving decomposition of $G$.

LEMMA 2.2. Let $G$ be a connected graph of order $n \geq 5$, let $T$ be a spanning tree of $G$ with $E(T)=\left\{e_{1}, e_{2}, \ldots, e_{n-1}\right\}$, and let $H=G-E(T)$. Then the decomposition $\mathscr{D}=\left\{F_{1}, F_{2}, \ldots, F_{n-1}, H\right\}$, where $E\left(F_{i}\right)=\left\{e_{i}\right\}$ for $1 \leq i \leq n-1$, is a resolving decomposition of $G$.

Proof. Let $e$ and $f$ be two edges of $G$. If $e$ and $f$ belong to distinct elements of $\mathscr{D}$, then $\mathcal{C}_{\mathscr{D}}(e) \neq \mathcal{C}_{\mathscr{D}}(f)$. Thus we may assume that $e$ and $f$ belong to the same element $H$ in $\mathscr{D}$. We show that $\mathcal{C}_{\mathscr{D}}(e) \neq \mathcal{C}_{\mathscr{D}}(f)$. Let $e=u v$, let $P$ be the unique $u-v$ path in $T$, and let $u^{\prime}$ and $v^{\prime}$ be the vertices on $P$ adjacent to $u$ and $v$, respectively. If $f$ is adjacent to at most one of $u u^{\prime}$ and $v v^{\prime}$, then either $d\left(e, u u^{\prime}\right) \neq d\left(f, u u^{\prime}\right)$ or $d\left(e, v v^{\prime}\right) \neq d\left(f, v v^{\prime}\right)$, and so $c_{\mathscr{D}}(e) \neq c_{\mathscr{D}}(f)$. Hence we may assume that $f$ is adjacent to both $u u^{\prime}$ and $v v^{\prime}$. We consider two cases according to whether $u^{\prime}=v^{\prime}$ or $u^{\prime} \neq v^{\prime}$.

CASE $1\left(u^{\prime}=v^{\prime}\right)$. Then $f$ is incident with the vertex $u^{\prime}$. Since $n \geq 5$ and $T$ is a spanning tree, there is a vertex $x \in V(G)-\left\{u, v, u^{\prime}\right\}$ such that $x$ is adjacent in $T$ with exactly one of $u, v$, and $u^{\prime}$. If $u^{\prime} x \in E(T)$, then $d\left(f, u^{\prime} x\right)=1 \neq$ $2=d\left(e, u^{\prime} x\right)$; otherwise, $d(e, u x)=1 \neq 2=d(f, u x)$ or $d(e, v x)=1 \neq 2=$ $d(f, v x)$ according to whether $u x$ or $v x$ is an edge of $T$. So $c_{\mathscr{D}}(e) \neq c_{\mathscr{D}}(f)$.

CASE $2\left(u^{\prime} \neq v^{\prime}\right)$. Then we may assume that $f$ is incident with $u^{\prime}$. Let $g$ be an edge of $T$ distinct from $u u^{\prime}$ that is incident with $u^{\prime}$. Then $d(e, g)=2 \neq 1=$ $d(f, g)$. Thus $\mathcal{C}_{\mathscr{D}}(e) \neq c_{\mathscr{D}}(f)$. 
We now present bounds for the resolving edge chromatic number of a connected graph in terms of its order and edge chromatic number.

THEOREM 2.3. If $G$ is a connected graph of order $n \geq 5$, then

$$
\chi_{e}(G) \leq \chi_{r e}(G) \leq n+\chi_{e}(G)-1 .
$$

PROoF. The lower bound follows by (1.4). To verify the upper bound, let $m$ be the size of $G$. If $G$ is a tree of order $n$, then $m=n-1$. Since $\chi_{r e}(G) \leq m$, the result is true for a tree. Thus we may assume that $G$ is a connected graph that is not a tree. Let $T$ be a spanning tree of $G$ with $E(T)=\left\{e_{1}, e_{2}, \ldots, e_{n-1}\right\}$. Let $H=$ $\langle E(G)-E(T)\rangle$ be the subgraph induced by $E(G)-E(T)$. Then $H$ is a nonempty subgraph of $G$. Let $\chi_{e}(H)=k$ and let $H_{1}, H_{2}, \ldots, H_{k}$ be the decomposition of $H$ into the color classes resulting from a minimum edge coloring of $H$. Now let

$$
\mathscr{D}=\left\{F_{1}, F_{2}, \ldots, F_{n-1}, H\right\}, \quad \mathscr{D}^{*}=\left\{F_{1}, F_{2}, \ldots, F_{n-1}, H_{1}, H_{2}, \ldots, H_{k}\right\},
$$

where $E\left(F_{i}\right)=\left\{e_{i}\right\}$ for $1 \leq i \leq n-1$. Since $\mathscr{D}$ is a resolving decomposition of $G$ by Lemma 2.2 and $D^{*}$ is a refinement of $\mathscr{D}$, it follows by Lemma 2.1 that $D^{*}$ is a resolving decomposition of $G$ as well. Thus $\mathscr{D}^{*}$ is a resolving independent decomposition of $G$, and so

$$
\chi_{r e}(G) \leq\left|\mathscr{D}^{*}\right|=n+k-1=n+\chi_{e}(H)-1 \leq n+\chi_{e}(G)-1,
$$

as desired.

Next, we present bounds for the resolving edge chromatic number of a connected graph in terms of its decomposition dimension and edge chromatic number.

THEOREM 2.4. For every connected graph $G$ of order at least 3,

$$
\operatorname{dim}_{d}(G) \leq \chi_{r e}(G) \leq \chi_{e}(G) \operatorname{dim}_{d}(G)
$$

Proof. By (1.4), it suffices to verify the upper bound: let $G$ be a nontrivial connected graph with $\operatorname{dim}_{d}(G)=k$ and $\chi_{e}(G)=c$. Furthermore, let $\mathscr{D}=$ $\left\{G_{1}, G_{2}, \ldots, G_{k}\right\}$ be a resolving decomposition of $G$. If $\mathscr{D}$ is independent, then $\mathscr{D}$ is a resolving independent decomposition of $G$ and so $\chi_{r e}(G) \leq|\mathscr{D}|=k=$ $\operatorname{dim}_{d}(G)<\chi_{e}(G) \operatorname{dim}_{d}(G)$ since $\chi_{e}(G) \geq 2$. Thus we may assume that $\mathscr{D}$ is not independent. Without loss of generality, assume that $E\left(G_{i}\right)$ is not independent in $E(G)$ for $1 \leq i \leq k_{1} \leq k$ and $E\left(G_{i}\right)$ is independent in $E(G)$ for $k_{1}+1 \leq i \leq k$ if $k_{1}<k$. Let $c_{i}=\chi_{e}\left(G_{i}\right)$ for $1 \leq i \leq k$ and so $1 \leq c_{i} \leq \chi_{e}(G)$. Define a decomposition $\mathscr{D}^{\prime}$ of $G$ from $\mathscr{D}$ by (1) decomposing each $G_{i}\left(1 \leq i \leq k_{1}\right)$ into $c_{i}$ color classes resulting from an edge coloring of $G_{i}$; (2) retaining each $G_{i}$ for $k_{1}+1 \leq i \leq k$. Certainly, $\mathscr{D}^{\prime}$ is an independent decomposition of $G$ with at most $\sum_{i=1}^{k} c_{i} \leq c k$ elements. Since $\mathscr{D}^{\prime}$ is a refinement of $\mathscr{D}$, it follows by virtue 
of Lemma 2.1 that $\mathscr{D}^{\prime}$ is also an independent resolving decomposition of $G$. Therefore, $\chi_{r e}(G) \leq\left|\mathscr{D}^{\prime}\right| \leq c k=\chi_{e}(G) \operatorname{dim}_{d}(G)$.

3. On resolving edge chromatic numbers of trees. The decomposition dimension of a tree $T$ was studied in [3, 6]. It was shown in [3] that $P_{n}$ is the only connected graph of order $n$ with decomposition dimension 2 . Although there is no general formula for the decomposition dimension of a nonpath tree, several bounds have been established for $\operatorname{dim}_{d}(T)$ for such trees in $[3,6]$. In this section, we investigate the resolving edge chromatic number of trees. Since $\chi_{r e}\left(P_{3}\right)=2$ and $\chi_{r e}\left(P_{n}\right)=3$ for $n \geq 4$, we consider trees that are not paths. First, we need some additional definitions and notation.

A vertex of degree at least 3 in a graph $G$ is called a major vertex. An endvertex $u$ of $G$ is said to be a terminal vertex of a major vertex $v$ of $G$ if $d(u, v)<$ $d(u, w)$ for every other major vertex $w$ of $G$. The terminal degree $\operatorname{ter}(v)$ of a major vertex $v$ is the number of terminal vertices of $v$. A major vertex $v$ of $G$ is an exterior major vertex of $G$ if it has positive terminal degree. Let $\sigma(G)$ denote the sum of the terminal degrees of the major vertices of $G$ and let $\operatorname{ex}(G)$ denote the number of exterior major vertices of $G$. In fact, $\sigma(G)$ is the number of end-vertices of $G$. For an ordered set $W=\left\{e_{1}, e_{2}, \ldots, e_{k}\right\}$ of edges in a connected graph $G$ and an edge $e$ of $G$, let

$$
c_{W}(e)=\left(d\left(e, e_{1}\right), d\left(e, e_{2}\right), \ldots, d\left(e, e_{k}\right)\right) .
$$

The following two results are useful to us, the first of which appeared in [9] and the second of which is due to König [8].

LEMMA 3.1. Let $T$ be a tree that is not a path, having order $n \geq 4$ and $p$ exterior major vertices $v_{1}, v_{2}, \ldots, v_{p}$. For $1 \leq i \leq p$, let $u_{i 1}, u_{i 2}, \ldots, u_{i k_{i}}$ be the terminal vertices of $v_{i}$, let $P_{i j}$ be the $v_{i}-u_{i j}$ path $\left(1 \leq j \leq k_{i}\right)$, and let $x_{i j}$ be a vertex in $P_{i j}$ that is adjacent to $v_{i}$. Let

$$
W=\left\{v_{i} x_{i j}: 1 \leq i \leq p, 2 \leq j \leq k_{i}\right\}
$$

Then $c_{W}(e) \neq c_{W}(f)$ for each pair $e, f$ of distinct edges of $T$ that are not edges of $P_{i j}$ for $1 \leq i \leq p$ and $2 \leq j \leq k_{i}$.

KÖNIG'S THEOREM. If $G$ is a bipartite graph, then $\chi_{e}(G)=\Delta(G)$. In particular, if $T$ is a tree, then $\chi_{e}(T)=\Delta(T)$.

For a cut-vertex $v$ in a connected graph $G$ and a component $H$ of $G-v$, the subgraph $H$ with the vertex $v$, together with all edges joining $v$ and $V(H)$ in $G$, is called a branch of $G$ at $v$. For a bridge $e$ in a connected graph $G$ and a component $F$ of $G-e$, the subgraph $F$, together with the bridge $e$, is called a branch of $G$ at $e$. For two edges $e=u_{1} u_{2}$ and $f=v_{1} v_{2}$ in $G$, an $e-f$ path in $G$ is a path with its initial edge $e$ and terminal edge $f$. 
We are now prepared to present an upper bound for the resolving edge chromatic number of a tree that is not a path.

THEOREM 3.2. Let $T$ be a tree that is not a path, having order $n \geq 4$ and $p$ exterior major vertices $v_{1}, v_{2}, \ldots, v_{p}$. For $1 \leq i \leq p$, let $u_{i 1}, u_{i 2}, \ldots, u_{i k_{i}}$ be the terminal vertices of $v_{i}$, let $P_{i j}$ be the $v_{i}-u_{i j}$ path $\left(1 \leq j \leq k_{i}\right)$, and let $x_{i j}$ be a vertex in $P_{i j}$ that is adjacent to $v_{i}$. Let $W$ be the set described in (3.2). Then

$$
\chi_{r e}(T) \leq \Delta(T-W)+\sigma(T)-\operatorname{ex}(T) .
$$

Proof. Let $U=\left\{v_{1}, u_{11}, u_{21}, \ldots, u_{p 1}\right\}$ and let $T_{0}$ be the subtree of $T$ of smallest size that contains $U$. For each pair $i, j$ of integers with $1 \leq i \leq p$ and $1 \leq j \leq k_{i}$, let $Q_{i j}=P_{i j}-v_{i}$ be the $x_{i j}-u_{i j}$ path in $T$. Thus $T-W$ is the union of the tree $T_{0}$ and the paths $Q_{i j}$ for all $i, j$ with $1 \leq i \leq p$ and $2 \leq j \leq k_{i}$. Since $T-W$ is a forest, it follows by König's theorem that $\chi_{e}(T-W)=\Delta(T-W)$. We define an edge coloring $c$ of $T$ by assigning (1) the colors to the edges in $T-W$ from the set $\{1,2, \ldots, \Delta(T-W)\}$; (2) the color

$$
c_{i j}=\Delta(T-W)+\left[k_{1}+k_{2}+\cdots+k_{i-1}-(i-1)\right]+(j-1)
$$

to the edge $v_{i} x_{i j}$ in $W$ for all $i, j$ with $1 \leq i \leq p$ and $2 \leq j \leq k_{i}$. Thus the maximum color assigned to the vertices of $G$ by $c$ is

$$
\begin{aligned}
c_{p, k_{p}} & =c\left(v_{p} x_{p, k_{p}}\right) \\
& =\Delta(T-W)+\left[k_{1}+k_{2}+\cdots+k_{p-1}-(p-1)\right]+\left(k_{p}-1\right) \\
& =\Delta(T-W)+\left(k_{1}+k_{2}+\cdots+k_{p}-p\right) \\
& =\Delta(T-W)+\sigma(T)-\operatorname{ex}(T) .
\end{aligned}
$$

Certainly, adjacent edges are colored differently by $c$ and so $c$ is an edge coloring of $T$. It remains to show that $c$ is a resolving edge coloring of $T$. Let

$$
k=\Delta(T-W)+\sigma(T)-\operatorname{ex}(T)
$$

and let $\mathscr{D}=\left\{C_{1}, C_{2}, \ldots, C_{k}\right\}$ be the decomposition of $G$ into the color classes resulting from $c$. Since all edges in $W$ are colored differently, it suffices to show that if $e, f \in E(T-W)$, then $\mathcal{C}_{\mathscr{D}}(e) \neq \mathcal{C}_{\mathscr{D}}(f)$. We consider three cases.

CASE $1\left(e, f \in E\left(T_{0}\right)\right)$. By Lemma 3.1, it follows that $c_{W}(e) \neq c_{W}(f)$, which implies that $c_{\mathscr{D}}(e) \neq \mathcal{C}_{\mathscr{D}}(f)$.

CASE $2\left(e, f \notin E\left(T_{0}\right)\right)$. There are two subcases.

SubCASE $2.1\left(e, f \in E\left(Q_{i j}\right)\right.$ for some $i, j$ with $1 \leq i \leq p$ and $\left.2 \leq j \leq k_{i}\right)$. Since $v_{i} x_{i j} \in W$ and $d\left(e, v_{i} x_{i j}\right) \neq d\left(f, v_{i} x_{i j}\right)$, this implies that $c_{W}(e) \neq c_{W}(f)$ and so $\mathcal{C}_{\mathscr{D}}(e) \neq \mathcal{C}_{\mathscr{D}}(f)$. 
SubCASE $2.2\left(e \in E\left(Q_{i j}\right)\right.$ and $f \in E\left(Q_{r s}\right)$, where $1 \leq i, r \leq p, 2 \leq j$, and $\left.s \leq k_{i}\right)$. Notice that if $i=r$, then $j \neq s$. Again, $v_{i} x_{i j}, v_{r} x_{r s} \in W$. If $d\left(e, v_{i} x_{i j}\right) \neq$ $d\left(f, v_{i} x_{i j}\right)$, then $c_{\mathscr{D}}(e) \neq c_{\mathscr{D}}(f)$. On the other hand, if $d\left(e, v_{i} x_{i j}\right)=d\left(f, v_{i} x_{i j}\right)$, then $d\left(f, v_{r} x_{r s}\right)<d\left(e, v_{r} x_{r s}\right)$, implying that $c_{\mathscr{D}}(e) \neq c_{\mathscr{D}}(f)$.

CASE 3 (exactly one of $e$ and $f$ belongs to $T_{0}$, say $f \in E\left(T_{0}\right)$ and $e \in E\left(Q_{i j}\right)$ for some $i, j$ with $1 \leq i \leq p$ and $\left.2 \leq j \leq k_{i}\right)$. If there is an edge $w \in W$ such that $f$ lies on the $e-w$ path, then $d(f, w)<d(e, w)$ and so $c_{\mathscr{D}}(e) \neq c_{\mathscr{D}}(f)$. Thus we may assume that every path between $e$ and any edge $w \in W$ does not contain $f$. Then $f$ lies on some path $P_{\ell 1}$ in $T$ for some $\ell$ with $1 \leq \ell \leq p$. We consider two subcases.

SubCASE $3.1(i=\ell)$. If $d\left(e, v_{i} x_{i j}\right) \neq d\left(f, v_{i} x_{i j}\right)$, then $\mathcal{C}_{\mathscr{D}}(e) \neq \mathcal{C}_{\mathscr{D}}(f)$. Thus we may assume that $d\left(e, v_{i} x_{i j}\right)=d\left(f, v_{i} x_{i j}\right)$. Since $v_{i}$ is an exterior vertex of $T$, it follows that $\operatorname{deg} v_{i} \geq 3$ and so there exists a branch $B$ at $v_{i}$ that does not contain $v_{i} x_{i j}$. Necessarily, $B$ must contain an edge $w$ of $W$. Then $d(f, w)<d(e, w)$ and so $\mathcal{C}_{\mathscr{D}}(e) \neq \mathcal{C}_{\mathscr{D}}(f)$.

SUBCASE $3.2(i \neq \ell)$. Since $v_{i}$ and $v_{\ell}$ are exterior major vertices, it follows that $\operatorname{deg} v_{i} \geq 3$ and $\operatorname{deg} v_{\ell} \geq 3$. Thus there exists a branch $B_{1}$ at $v_{i}$ that does not contain $v_{i} x_{i j}$ and a branch $B_{2}$ at $v_{\ell}$ that does not contain $v_{\ell} x_{\ell 1}$. Necessarily, each of $B_{1}$ and $B_{2}$ must contain an edge of $W$. Let $w_{1}$ and $w_{2}$ be two edges of $T$ such that $w_{i}$ belongs to $B_{i}$ for $i=1,2$. If $d\left(e, w_{2}\right) \neq d\left(f, w_{2}\right)$, then $c_{W}(e) \neq c_{W}(f)$ and so $c_{\mathscr{D}}(e) \neq c_{\mathscr{D}}(f)$. Thus we may assume that $d\left(e, w_{2}\right)=$ $d\left(f, w_{2}\right)$. However, then, $d\left(e, w_{1}\right)<d\left(f, w_{1}\right)$, implying that $c_{W}(e) \neq c_{W}(f)$ and so $\mathcal{C}_{\mathscr{D}}(e) \neq \mathcal{C}_{\mathscr{D}}(f)$.

Thus, in any case, $\mathcal{C}_{\mathscr{D}}(e) \neq \mathcal{C}_{\mathscr{D}}(f)$ and so $\mathscr{D}$ is a resolving edge coloring of $G$. Therefore, $\chi_{r e}(T) \leq \Delta(T-W)+\sigma(T)-\operatorname{ex}(T)$.

The upper bound in Theorem 3.2 is sharp. To see this, let $K_{1, n}, n \geq 3$, be the star with $V\left(K_{1, n}\right)=\left\{v, v_{1}, v_{2}, \ldots, v_{n}\right\}$, where $v$ is the central vertex of $K_{1, n}$, and let $T$ be the tree obtained from $K_{1, n}$ by subdividing each edge $v v_{i}$ into $v x_{i}$ and $x_{i} v_{i}$ for $2 \leq i \leq n$. Let $W=\left\{v x_{i}: 2 \leq i \leq n\right\}$. Then it can be verified that $x_{r e}(T)=\Delta(T-W)+\sigma(T)-\operatorname{ex}(T)=n$.

Next, we present another upper bound for $\chi_{r e}(T)$ in terms of the maximum degree of a tree $T$. A major vertex of a tree $T$ is a superior major vertex of $T$ if its terminal degree is at least 2. Let $\sup (T)$ denote the number of superior major vertices of $T$. Thus every superior major vertex of $T$ is also an exterior major vertex. Hence, if $T$ is a tree that is not a path, then $1 \leq \sup (T) \leq \operatorname{ex}(T)$.

THEOREM 3.3. If $T$ is a tree that is not a path, then

$$
\chi_{r e}(T) \leq \Delta(T)+\sup (T) .
$$

PROoF. Suppose that $T$ contains $q \geq 1$ superior major vertices $v_{1}, v_{2}, \ldots, v_{q}$. For $1 \leq i \leq q$, let $u_{i 1}, u_{i 2}, \ldots, u_{i k_{i}}$ be the terminal vertices of $v_{i}$, where $k_{i} \geq 2$. For each $i$, $j$ with $1 \leq i \leq q$ and $1 \leq j \leq k_{i}$, let $P_{i j}$ be the $v_{i}-u_{i j}$ path in $T$, 
let $x_{i j}$ be the vertex in $P_{i j}$ that is adjacent to $v_{i}$, and let $Q_{i j}=P_{i j}-v_{i}$ be the $x_{i j}-u_{i j}$ path in $T$. Furthermore, let

$$
W^{*}=\left\{v_{i} x_{i 2}: 1 \leq i \leq q\right\}
$$

and let $T_{1}$ be the subgraph of $T$ obtained by removing all vertices in each set $V\left(Q_{i j}\right)-\left\{x_{i j}\right\}$ from $T$ for all $i, j$ with $1 \leq i \leq q$ and $1 \leq j \leq k_{i}$; that is,

$$
T_{1}=T-\left(\cup\left\{V\left(Q_{i j}\right)-\left\{x_{i j}\right\}: 1 \leq i \leq q, 1 \leq j \leq k_{i}\right\}\right) .
$$

Let $Q$ be the linear forest whose components are the paths $Q_{i j}(1 \leq i \leq q$ and $\left.1 \leq j \leq k_{i}\right)$ in $T$; that is,

$$
Q=\cup\left\{Q_{i j}: 1 \leq i \leq q, 1 \leq j \leq k_{i}\right\}
$$

Let

$$
T_{0}=T_{1}-\left\{x_{i 2}: 1 \leq i \leq q\right\} .
$$

Then $E\left(T_{0}\right)=E\left(T_{1}\right)-W^{*}$ and

$$
E(T)=E\left(T_{0}\right) \cup W^{*} \cup E(Q)
$$

Hence $E(T)$ is partitioned into $E\left(T_{0}\right), W^{*}$, and $E(Q)$. We define an edge coloring $c$ of $T$ by coloring the edges in each of the sets $E\left(T_{0}\right), W^{*}$, and $E(Q)$ in the following three steps:

(1) if $T$ has only one exterior major vertex, then this exterior major vertex is also a superior major vertex since $T$ is not a path. Thus $\Delta\left(T_{0}\right)=\Delta(T)-1$ and so $\chi_{e}\left(T_{0}\right)=\Delta(T)-1$. Let $c_{1}$ be an edge coloring of $T_{0}$ using $\Delta(T)-1$ colors and define $c(e)=c_{1}(e)$ for all $e \in E\left(T_{0}\right)$. If $T$ has more than one exterior major vertex, then $\Delta\left(T_{0}\right) \leq \Delta(T)$ and so $\chi_{e}\left(T_{0}\right) \leq \Delta(T)$. Let $c_{1}^{\prime}$ be an edge coloring of $T_{0}$ using $\Delta(T)$ colors and define $c(e)=c_{1}^{\prime}(e)$ for all $e \in E\left(T_{0}\right)$;

(2) define $c\left(v_{i} x_{i 2}\right)=\Delta(T)+i$ for each edge $v_{i} x_{i 2}$ in $W^{*}$, where $1 \leq i \leq q$;

(3) define $c(e)$ for each edge $e$ in $Q$. For each pair $i, j$ with $1 \leq i \leq q$ and $1 \leq j \leq k_{i}$, let $m_{i j}=\left|E\left(Q_{i j}\right)\right|$ and

$$
E\left(Q_{i j}\right)=\left\{e_{i j}^{1}, e_{i j}^{2}, \ldots, e_{i j}^{m_{i j}}\right\},
$$

where (1) $e_{i j}^{1}$ is incident with $x_{i j}$, (2) $e_{i j}^{m_{i j}}$ is incident with $u_{i j}$, (3) $e_{i j}^{s}$ is adjacent to $e_{i j}^{s+1}$ in $Q_{i j}$ for all $s$ with $1 \leq s \leq m_{i j}-1$. Let

$$
T_{0}^{*}=T_{1}-\left\{x_{i j}: 1 \leq i \leq q, 1 \leq j \leq k_{i}\right\} .
$$


For each $i$ with $1 \leq i \leq q$, let $d_{i}=\operatorname{deg}_{T_{0}^{*}} v_{i}$, and so the degree of $v_{i}$ in $T$ is

$$
\operatorname{deg} v_{i}=d_{i}+k_{i} \leq \Delta(T)
$$

We consider two cases according to whether $d_{i}=0$ or $d_{i}>0$.

CASE $1\left(d_{i}=0\right)$. Thus $N_{T_{0}^{*}}\left(v_{i}\right)=\varnothing$. This implies that $T$ has only one exterior major vertex that is also a superior major vertex. Notice that if $j_{1}, j_{2} \in$ $\left\{1,3,4, \ldots, k_{1}\right\}$ and $j_{1} \neq j_{2}$, then $v_{1} x_{1 j_{1}}$ and $v_{1} x_{1 j_{2}}$ are adjacent edges in $T_{0}$ and so $c\left(v_{1} x_{1 j_{1}}\right) \neq c\left(v_{1} x_{1 j_{2}}\right)$. There are two subcases.

SubCASE $1.1\left(k_{1}=3\right)$. Define

$$
\begin{aligned}
& c\left(e_{11}^{s}\right)=c\left(v_{1} x_{13}\right) \quad \text { if } s \text { is odd, } 1 \leq s \leq m_{11}, \\
& c\left(e_{11}^{s}\right)=c\left(v_{1} x_{11}\right) \quad \text { if } s \text { is even, } 2 \leq s \leq m_{11}, \\
& c\left(e_{12}^{s}\right)=\Delta(T) \text { if } s \text { is odd, } 1 \leq s \leq m_{12}, \\
& c\left(e_{12}^{s}\right)=c\left(v_{1} x_{11}\right) \quad \text { if } s \text { is even, } 2 \leq s \leq m_{12}, \\
& c\left(e_{13}^{s}\right)=\Delta(T) \text { if } s \text { is odd, } 1 \leq s \leq m_{13}, \\
& c\left(e_{13}^{s}\right)=c\left(v_{1} x_{13}\right) \quad \text { if } s \text { is even, } 2 \leq s \leq m_{13} .
\end{aligned}
$$

SubCASE $1.2\left(k_{1} \geq 4\right)$. For $s$ is even and $2 \leq s \leq m_{11}$, define $c\left(e_{11}^{s}\right)$ as in (3.17); for $1 \leq s \leq m_{12}$, define $c\left(e_{12}^{s}\right)$ as in (3.18); for $1 \leq s \leq m_{13}$, define $c\left(e_{13}^{s}\right)$ as in (3.19). Furthermore, define

$$
\begin{aligned}
& c\left(e_{11}^{s}\right)=c\left(v_{1} x_{1 k_{1}}\right) \quad \text { if } s \text { is odd, } 1 \leq s \leq m_{11}, \\
& c\left(e_{1 j}^{s}\right)=c\left(v_{1} x_{1, j-1}\right) \quad \text { if } s \text { is odd, } 1 \leq s \leq m_{1 j}, 4 \leq j \leq k_{1}, \\
& c\left(e_{1 j}^{s}\right)=c\left(v_{1} x_{1 j}\right) \text { if } s \text { is even, } 2 \leq s \leq m_{1 j}, 4 \leq j \leq k_{1} .
\end{aligned}
$$

CASE $2\left(d_{i}>0\right)$. Thus $N_{T_{0}^{*}}\left(v_{i}\right) \neq \varnothing$. Let $x \in N_{T_{0}^{*}}\left(v_{i}\right)$. Then $v_{i} x$ and $v_{i} x_{i j}$ $\left(1 \leq j \leq k_{1}\right)$ are adjacent edges in $T_{0}$ and so all colors $c\left(v_{i} x\right)$ and $c\left(v_{i} x_{i j}\right)$, $1 \leq j \leq k_{1}$, are distinct. There are three subcases.

SubCASE $2.1\left(k_{i}=2\right)$. Define

$$
\begin{aligned}
& c\left(e_{i 1}^{s}\right)=c\left(v_{i} x\right) \quad \text { if } s \text { is odd, } 1 \leq s \leq m_{i 1}, \\
& c\left(e_{i 1}^{s}\right)=c\left(v_{i} x_{i 1}\right) \quad \text { if } s \text { is even, } 2 \leq s \leq m_{i 1}, \\
& c\left(e_{i 2}^{s}\right)=c\left(v_{i} x\right) \quad \text { if } s \text { is odd, } 1 \leq s \leq m_{i 2}, \\
& c\left(e_{i 2}^{s}\right)=c\left(v_{i} x_{i 1}\right) \quad \text { if } s \text { is even, } 2 \leq s \leq m_{i 2} .
\end{aligned}
$$

SubCASE $2.2\left(k_{i}=3\right)$. For $s$ is even and $2 \leq s \leq m_{i 1}$, define $c\left(e_{i 1}^{s}\right)$ as in (3.22); for $1 \leq s \leq m_{i 2}$, define $c\left(e_{i 2}^{s}\right)$ as in (3.23), and define

$$
\begin{aligned}
& c\left(e_{i 1}^{s}\right)=c\left(v_{i} x_{i 3}\right) \quad \text { if } s \text { is odd, } 1 \leq s \leq m_{i 1}, \\
& c\left(e_{i 3}^{s}\right)=c\left(v_{i} x\right) \quad \text { if } s \text { is odd, } 1 \leq s \leq m_{i 3}, \\
& c\left(e_{i 3}^{s}\right)=c\left(v_{i} x_{i 3}\right) \quad \text { if } s \text { is even, } 2 \leq s \leq m_{i 3} .
\end{aligned}
$$


SubCASE $2.3\left(k_{i} \geq 4\right)$. For $s$ is even and $2 \leq s \leq m_{i 1}$, define $c\left(e_{i 1}^{s}\right)$ as in (3.22); for $1 \leq s \leq m_{i 2}$, define $c\left(e_{i 2}^{s}\right)$ as in (3.23); for $1 \leq s \leq m_{i 3}$, define $c\left(e_{i 3}^{s}\right)$ as in (3.25). Furthermore, define

$$
\begin{aligned}
& c\left(e_{i 1}^{s}\right)=c\left(v_{i} x_{i k_{i}}\right) \quad \text { if } s \text { is odd, } 1 \leq s \leq m_{i 1}, \\
& c\left(e_{i j}^{s}\right)=c\left(v_{i} x_{i, j-1}\right) \quad \text { if } s \text { is odd, } 1 \leq s \leq m_{i j}, 4 \leq j \leq k_{i}, \\
& c\left(e_{i j}^{s}\right)=c\left(v_{i} x_{i j}\right) \text { if } s \text { is even, } 2 \leq s \leq m_{i j}, 4 \leq j \leq k_{i} .
\end{aligned}
$$

Since adjacent edges of $T$ are colored differently by $c$, it follows that $c$ is an edge coloring of $T$ using $\Delta(T)+q$ colors. It remains to show that $c$ is a resolving edge coloring of $T$. Let $\mathscr{D}=\left\{C_{1}, C_{2}, \ldots, C_{\Delta(T)+q}\right\}$ be the decomposition of $T$ into the color classes of $c$. Since all edges in $W^{*}$ are colored differently by $c$, it suffices to show that if $e, f \in E\left(T-W^{*}\right)$, then $c_{\mathscr{D}}(e) \neq c_{\mathscr{D}}(f)$. We consider two cases.

CASE 1 (there is some exterior major vertex $z$ of $T$ and a terminal vertex $x$ of $z$ such that $e$ lies on the $z-x$ path of $T$ ). Let $y$ be a vertex in the $z-x$ path that is adjacent to $z$. There are two subcases.

SubCASE 1(a) $(y z \in W)$. First, assume that $f$ lies on some $z-x^{*}$ path of $T$ for some terminal vertex $x^{*}$ of $z$. If $x=x^{*}$, then either $d(e, y z)<d(f, y z)$ or $d(f, y z)<d(e, y z)$, implying that $c_{\mathscr{D}}(e) \neq c_{\mathscr{D}}(f)$. Thus we may assume that $x \neq x^{*}$. If $d(e, y z) \neq d(f, y z)$, then $c_{\mathscr{D}}(e) \neq c_{\mathscr{D}}(f)$. If $d(e, y z)=d(f, y z)$, then $c(e) \neq c(f)$ by the definition of $c$ and so $c_{\mathscr{D}}(e) \neq \mathcal{C}_{\mathscr{D}}(f)$.

Next, assume that $f$ does not lie on any $z-x^{*}$ path of $T$ for all terminal vertices $x^{*}$ of $z$. If there is an edge $w \in W^{*}$ such that either $f$ lies on the $e-w$ path or $e$ lies on the $f-w$ path, then $d(f, w)<d(e, w)$ or $d(e, w)<d(f, w)$, respectively. In either case, $c_{\mathscr{D}}(e) \neq c_{\mathscr{D}}(f)$. Thus, we may assume that every path between $e$ and an edge of $W^{*}$ does not contain $f$ and every path between $f$ and an edge of $W^{*}$ does not contain $e$. Necessarily, then, there exist an exterior major vertex $z^{\prime}$ and a terminal vertex $x^{\prime}$ of $z^{\prime}$ such that $f$ lies on the $z^{\prime}-x^{\prime}$ path of $T$. Since $f$ does not lie on any $z-x^{*}$ path of $T$ for all terminal vertices $x^{*}$ of $z$, it follows that $z \neq z^{\prime}$. Since $z^{\prime}$ is an exterior major vertex of $T$, it follows that the degree of $z^{\prime}$ is at least 3 and so there exists a branch $B$ at $z^{\prime}$ that does not contain $f$. Necessarily, $B$ must contain an edge of $W^{*}$. Let $w^{*}$ be an edge of $W^{*}$ that belongs to $B$. If $d(e, y z) \neq d(f, y z)$, then $c_{\mathscr{D}}(e) \neq \mathcal{C}_{\mathscr{D}}(f)$. Thus we may assume that $d(e, y z)=d(f, y z)$. This implies that $d\left(f, w^{*}\right)<d\left(e, w^{*}\right)$ and so $\mathcal{C}_{\mathscr{D}}(e) \neq \mathcal{C}_{\mathscr{D}}(f)$.

SubCASE 1(b) $(y z \notin W)$. By the argument used in Subcase 1.1, we may assume that every path between $e$ and an edge of $W^{*}$ does not contain $f$ and every path between $f$ and an edge of $W^{*}$ does not contain $e$. Thus there exist an exterior major vertex $z^{\prime}$ and a terminal vertex $x^{\prime}$ of $z^{\prime}$ such that $f$ lies on the $z^{\prime}-x^{\prime}$ path of $T$. If $z=z^{\prime}$, then there exists $w \in W^{*}$ such that $w$ is incident with $z$. If $d(e, w) \neq d(f, w)$, then $c_{\mathscr{D}}(e) \neq c_{\mathscr{D}}(f)$, while if $d(e, w)=d(f, w)$, then $c(e) \neq c(f)$ by the definition of $c$ and so $c_{\mathscr{D}}(e) \neq c_{\mathscr{D}}(f)$. Thus we may 
assume that $z \neq z^{\prime}$. Since the degrees of $z$ and $z^{\prime}$ are at least 3 , there exists a branch $B_{1}$ at $z$ that does not contain $e$ and a branch $B_{2}$ at $z^{\prime}$ that does not contain $f$. Necessarily, $B_{1}$ must contain an edge $w_{1}$ of $W^{*}$ and $B_{2}$ must contain an edge $w_{2}$ of $W^{*}$. If $d\left(e, w_{1}\right) \neq d\left(f, w_{1}\right)$, then $\mathcal{C}_{\mathscr{D}}(e) \neq \mathcal{c}_{\mathscr{D}}(f)$, while if $d\left(e, w_{1}\right)=d\left(f, w_{1}\right)$, then $d\left(f, w_{2}\right)<d\left(e, w_{2}\right)$ and so $c_{\mathscr{D}}(e) \neq \mathcal{C}_{\mathscr{D}}(f)$.

CASE 2 (for every exterior major vertex $z$ of $T$ and every terminal vertex $x$ of $z$, $e$ does not lie on the $z-x$ path of $T$ ). Then there are at least two branches at $e$, say $B_{1}^{\prime}$ and $B_{2}^{\prime}$, each of which contains some superior major vertex. Therefore, each of $B_{1}^{\prime}$ and $B_{2}^{\prime}$ contains an edge of $W^{*}$. Let $w_{1}^{\prime}$ and $w_{2}^{\prime}$ be the edges of $W^{*}$ in $B_{1}^{\prime}$ and $B_{2}^{\prime}$, respectively. First assume that $f \in E\left(B_{1}^{\prime}\right)$. Then the $f-w_{2}^{\prime}$ path of $T$ contains $e$, so $d\left(e, w_{2}^{\prime}\right)<d\left(f, w_{2}^{\prime}\right)$ and $c_{\mathscr{D}}(e) \neq \mathcal{C}_{\mathscr{D}}(f)$. We now assume that $f \notin E\left(B_{1}^{\prime}\right)$. Then the $f-w_{1}^{\prime}$ path of $T$ contains $e$. Hence $d\left(e, w_{1}^{\prime}\right)<d\left(f, w_{1}^{\prime}\right)$, so $c_{\mathscr{D}}(e) \neq c_{\mathscr{D}}(f)$.

Therefore, $\mathscr{D}$ is a resolving edge coloring of $T$ and so $\chi_{r e}(T) \leq|\mathscr{D}|=\Delta(T)+$ $\sup (T)$, as desired.

In the proof of Theorem 3.3, if $T$ is a tree with $\sup (T) \geq 2 \operatorname{such}$ that $\operatorname{deg} v \leq$ $\Delta(T)-1$ for every major vertex $v$ of $T$ that is not a superior major vertex, then $\Delta\left(T_{0}\right) \leq \Delta(T)-1$. Hence $\chi_{e}\left(T_{0}\right) \leq \Delta(T)-1$. Thus, $T_{0}$ has an edge coloring $c^{*}$ using $\Delta(T)-1$ colors. Define an edge coloring $c$ such that $c(e)=c^{*}(e)$ for all $e \in E\left(T_{0}\right)$ and define $c(e)$ for each $e \in V(T)-E\left(T_{0}\right)$ as described in the proof of Theorem 3.3. Then an argument similar to the one used in the proof of Theorem 3.3 shows that $c$ is a resolving edge coloring of $T$. Thus, we have the following corollary.

COROLlARY 3.4. Let $T$ be a tree with $\sup (T) \geq 2$. If every major vertex $v$ of $T$ that is not a superior major vertex has $\operatorname{deg} v<\Delta(T)$, then

$$
\chi_{r e}(T) \leq \Delta(T)+\sup (T)-1
$$

The upper bound in Corollary 3.4 is sharp. To see this, let $T$ be a tree having two superior major vertices $v_{1}$ and $v_{2}$ with $\operatorname{deg} v_{1}=\operatorname{deg} v_{2}=\Delta(T)$ and $\operatorname{deg} v<$ $\Delta(T)$ for every major vertex $v$ of $T$ that is not a superior major vertex. By Corollary $3.4, \chi_{r e}(T) \leq \Delta(T)+\sup (T)-1=\Delta(T)+1$. Assume, to the contrary, that $\chi_{r e}(T)=\Delta(T)$. Let $c$ be a resolving edge coloring of $T$ with $\Delta(T)$ colors and let $\mathscr{D}=\left\{C_{1}, C_{2}, \ldots, C_{\Delta(T)}\right\}$ be the decomposition of $T$ into the color classes of $c$. Let $N\left(v_{i}\right)=\left\{x_{i 1}, x_{i 2}, \ldots, x_{i \Delta(T)}\right\}$ for $i=1,2$. Without loss of generality, assume that $x_{i j} \in C_{j}$ for $i=1,2$ and $1 \leq j \leq \Delta(T)$. However, then, $c_{\mathscr{D}}\left(v_{1} x_{11}\right)=$ $(0,1,1, \ldots)=c_{\mathscr{D}}\left(v_{2} x_{21}\right)$, which is a contradiction. Therefore, $\chi_{r e}(T)=\Delta(T)+$ $1=\Delta(T)+\sup (T)-1$.

ACKNOWLEDGments. We are grateful to Professor Gary Chartrand for suggesting problems on resolving edge colorings of graphs and kindly providing useful information on this topic. Also, we thank Professor Peter Slater for the 
useful conversation. This research was supported in part by a Western Michigan University Faculty Research and Creative Activities Fund.

\section{REFERENCES}

[1] G. Chartrand, D. Erwin, M. A. Henning, P. J. Slater, and P. Zhang, The locatingchromatic number of a graph, Bull. Inst. Combin. Appl. 36 (2002), 89-101.

[2] _ Graphs of order $n$ with locating-chromatic number $n-1$, Discrete Math. 269 (2003), 65-79.

[3] G. Chartrand, D. Erwin, M. Raines, and P. Zhang, The decomposition dimension of graphs, Graphs Combin. 17 (2001), no. 4, 599-605.

[4] G. Chartrand and L. Lesniak, Graphs \& Digraphs, 3rd ed., Chapman \& Hall, London, 1996.

[5] G. Chartrand, V. Saenpholphat, and P. Zhang, Resolving edge colorings in graphs, to appear in Ars Combin.

[6] H. Enomoto and T. Nakamigawa, On the decomposition dimension of trees, Discrete Math. 252 (2002), no. 1-3, 219-225.

[7] F. Harary and R. A. Melter, On the metric dimension of a graph, Ars Combin. 2 (1976), 191-195.

[8] D. König, Über Graphen und ihre anwendung auf Determinantentheorie und Mengenlehre, Math. Ann. 77 (1916), 453-465 (German).

[9] V. Saenpholphat and P. Zhang, Connected resolving decompositions in graphs, to appear in Math. Bohem.

[10] - On connected resolving decompositions in graphs, to appear in Czechoslovak Math. J.

[11] P. J. Slater, Leaves of trees, Proceedings of the 6th Southeastern Conference on Combinatorics, Graph Theory, and Computing (Florida Atlantic Univ., Boca Raton, Fla, 1975), Congressus Numerantium, vol. 14, Utilitas Mathematica, Manitoba, 1975, pp. 549-559.

[12] _ Dominating and reference sets in a graph, J. Math. Phys. Sci. 22 (1988), no. $4,445-455$.

Varaporn Saenpholphat: Mathematics Department, Srinakharinwirot University, Sukhumvit 23, Bangkok 10110, Thailand

E-mail address: varaporn@swu.ac.th

Ping Zhang: Department of Mathematics, Western Michigan University, Kalamazoo, MI 49008, USA

E-mail address: ping. zhang@wmich .edu 


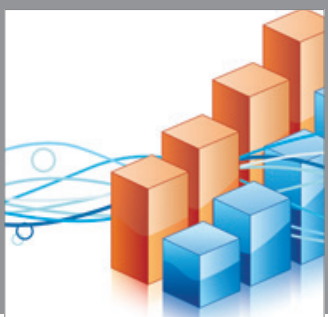

Advances in

Operations Research

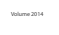

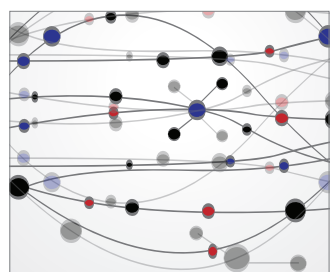

\section{The Scientific} World Journal
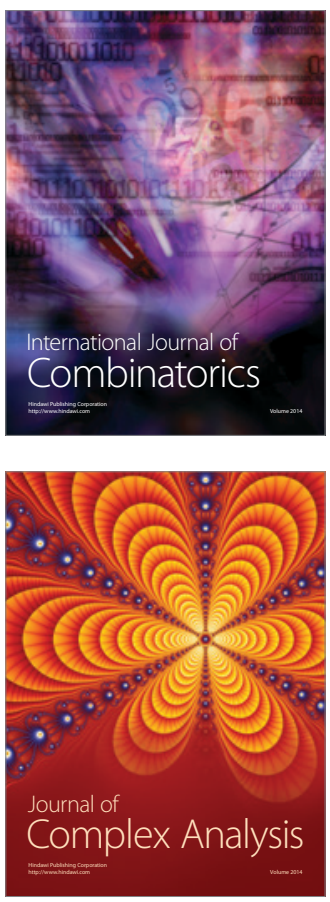

International Journal of

Mathematics and

Mathematical

Sciences
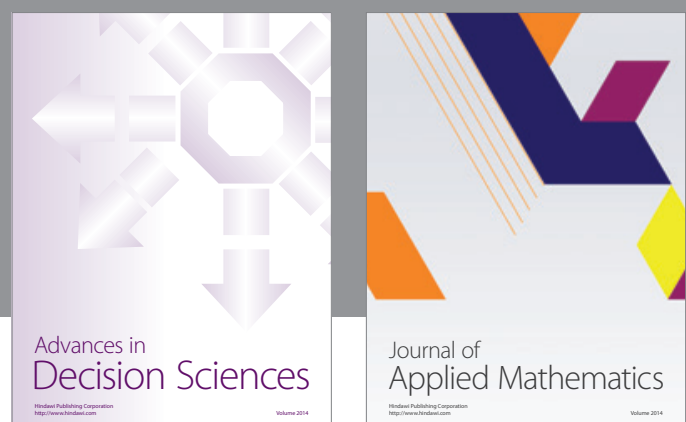

Journal of

Applied Mathematics
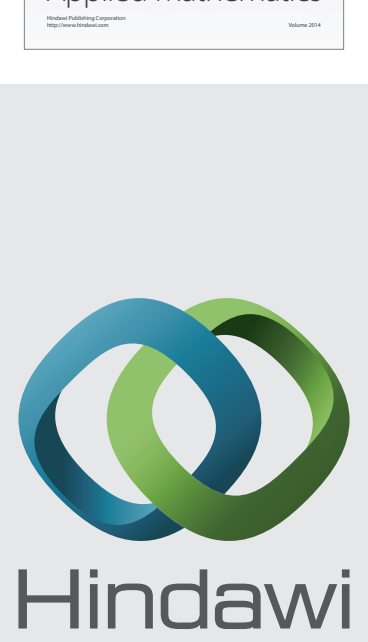

Submit your manuscripts at http://www.hindawi.com
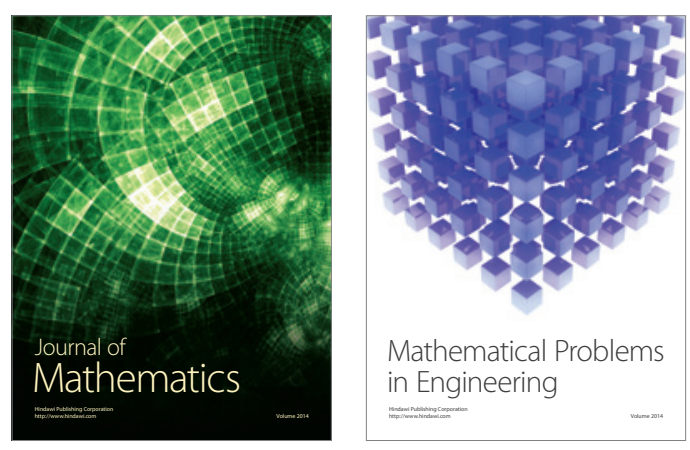

Mathematical Problems in Engineering
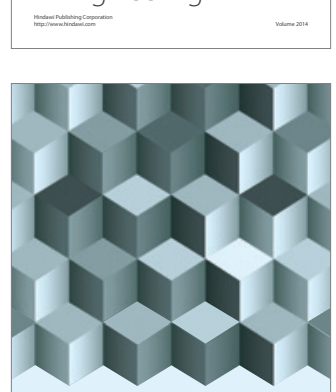

Journal of

Function Spaces
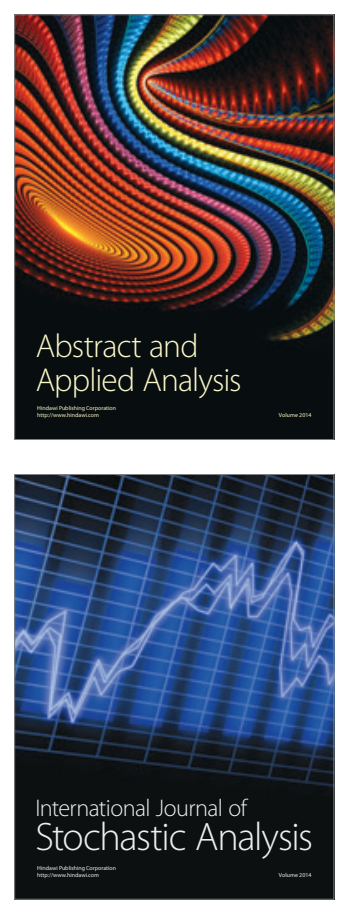

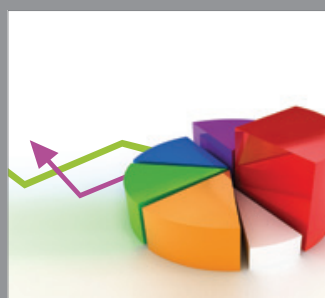

ournal of

Probability and Statistics

Promensencen
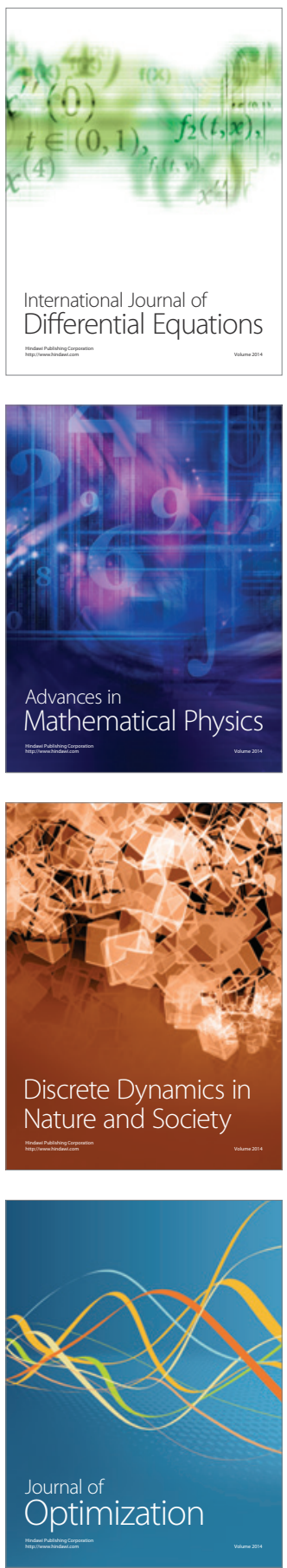